SCIENCE \& TECHNOLOGY
Journal homepage: http://www.pertanika.upm.edu.my/

\title{
Assessment by Simulation of Different Topological Integration of Solar Photovoltaic Plant in Medium Voltage Distribution Networks
}

\author{
Md. Milon Uddin*, Mushfiqur Rahman, Md. Tanzid Ridwan Hossain and \\ Md. Habibur Rahman
}

Institute of Energy, University of Dhaka, Dhaka-1000, Bangladesh

\begin{abstract}
Fossil fuels are diminishing day by day and are being utilized for various purposes like transportation, electricity generation, cooking and other uses which emits harmful gases. Global warming is increasing due to greenhouse gases. Sunlight is abundant compared to fossil fuels. Photovoltaic (PV) power plant could be one of the best techniques to lessen using of fossil fuels. Effect of different topological integration of solar photovoltaic plant in medium voltage distribution network had been analyzed in this research. Analysis had been done based on 1. loadability (capability of an electrical grid to run the connected load) 2. maximum power input. Fractioning (a way of splitting the total photovoltaic power generation into different size and number of plant) generation effect of PV generators in distribution systems had been found by a comparative study. Results show that change in loadability was due to placement and fraction of solar power plant in various buses. In addition, maximum input power of PV system varied with the placement of generation units among picked buses. Interaction among PV systems, and induction machine had been carried out and the result shows that interaction among PV and induction machine was different in terms of loadability because of fractioning. It had been found that change of loadability occurred due to fractioning and distribution of photovoltaic systems on

different types of designed topology.
\end{abstract}

ARTICLE INFO

Article history:

Received: 05 September 2020

Accepted: 16 February 2021

Published: 30 April 2021

DOI: https://doi.org/10.47836/pjst.29.2.25

E-mail addresses:

milon.brac456@gmail.com (Md. Milon Uddin)

mushfiqbd91@gmail.com (Mushfiqur Rahman)

tanzid_ridwan@yahoo.com (Md. Tanzid Ridwan Hossain)

mhabib@du.ac.bd (Md. Habibur Rahman)

* Corresponding author
Keywords: Fossil fuel, greenhouse gases (GHG), loadability, medium voltage (MV), Photovoltaic (PV)

\section{INTRODUCTION}

Loadability is the capability of an electrical grid to run the connected load. Effect of integration of same size photovoltaic 
generation plant into different buses of electrical medium voltage distribution network may vary in terms of lodability. A feasibility study using photovoltaic (PV), as an alternate source of electricity generation in Bangladesh, was done by analyzing the stability of electrical power systems with the penetration of photovoltaic based generation. Systemloading margin was studied without and with PV based generator. The contribution of PV based generator on solving under voltage problem and improving bus voltage was studied. Solution of overloading problem of power transformers with solar PV generator was described (Khan et al., 2013). A study has been done about optimal placement and sizing method to improve the voltage stability margin in a distribution system using distributed generation (DG). In this study it had been observed that operating condition of Distributed generation (DG) affected the sizing and placing when the distributed generation units operated at the unity power factor. If it is necessary, they need to put in the most impressionable voltage buses to ameliorate the stability margin of voltage (Al Abri et al., 2013). A study was done for site selection of plug in hybrid vehicle (PHEV) charging station based on voltage sensitivity of commercial distribution system. The analysis was done in a 16-bus system in power factory simulation software. Voltage sensitivity factor was used to find an optimal place for the PHEV charging station (Rahman et al., 2013).

Voltage stability is an important part of power system stability. Voltage instability is occurred due to lack of reactive power supplied to the electrical grid. Definition of voltage stability is given by IEEE power system engineering committee in the following way (Begovic et al., 1995). Voltage stability is the ability of a system to maintain voltage so that when load admittance increased, load power will increase, and voltages are controllable.

The definition of power system stability given by IEEEICIGER task force is "Power system stability is an ability of an electric power system, for a given initial operating condition, to regain a state of operating equilibrium after being subjected to a physical disturbance, with most system variables bounded so that practically the entire system remains intact" (Ingelsson et al., 1997).

The impact of Distributed Generation (DG) technology and penetration level on the dynamics of a test system has been presented in a research. From this research it has been found that effects of distributed generations on the dynamics of a power system depend on the technology of the Distributed Generators (Stootweg et al, 2002). A practical investigation of the impacts of Distributed Generation (DG) units on system stability has been studied on a Brazilian real network. It was found that the distribution generation enhanced the overall performance (Londero et al., 2009). A research showed an assessment of the impact of the Distribution Generation unit size and location under a change in the loading conditions due to a contingency on unbalanced distribution systems (Kotamarty et al., 2008). A study has analyzed the potential impacts of Distributed Generation (DG) on the stability on the electrical networks. The investigation was carried out at a constant load 
demands but with different contributions from fuel cells and micro-turbines. As a result, the rated and supplied powers of the traditional synchronous generators were adjusted to gain the power balance in the network (Azmy \& Erlich, 2005)

The static voltage stability impact of solar photovoltaic generation on power networks using PowerWorld simulator power-voltage (P-V) and voltage-reactive (V-Q) carves to investigate the renewable energy generator model performance suitability has been examined. Form the simulation result, it has been recommended that in a steady-state analysis of the grid power system the effects of power factor (pf) and voltage drop control should be considered by power grid engineers (Muhammed \& Rawa, 2020).

Basic phenomenon behind voltage stability:

- Voltage level decreases with loading

- No increase of voltage with addition of a new generation unit if the system reaches to its maximum transfer limit

- High reactive loading causes reduction of voltage in that area

- Load quality has an effect on voltage change

- Transfer capacity is reduced due to reactive loading

It is important to study the V-P (voltage vs power) curve for analyzing the voltage level stability of a power distribution system. Greater the output power from photovoltaic or solar thermal greater the load ability of a specific grid system. A sample P-V curve has been showed in the Figure 1.

Load quality has an effect on loadability. The part below the critical voltage in the curve cannot be found from practical system operation. This part is mathematical expression. In this condition collapse of voltage occurs in a grid. In case of higher load demand, control

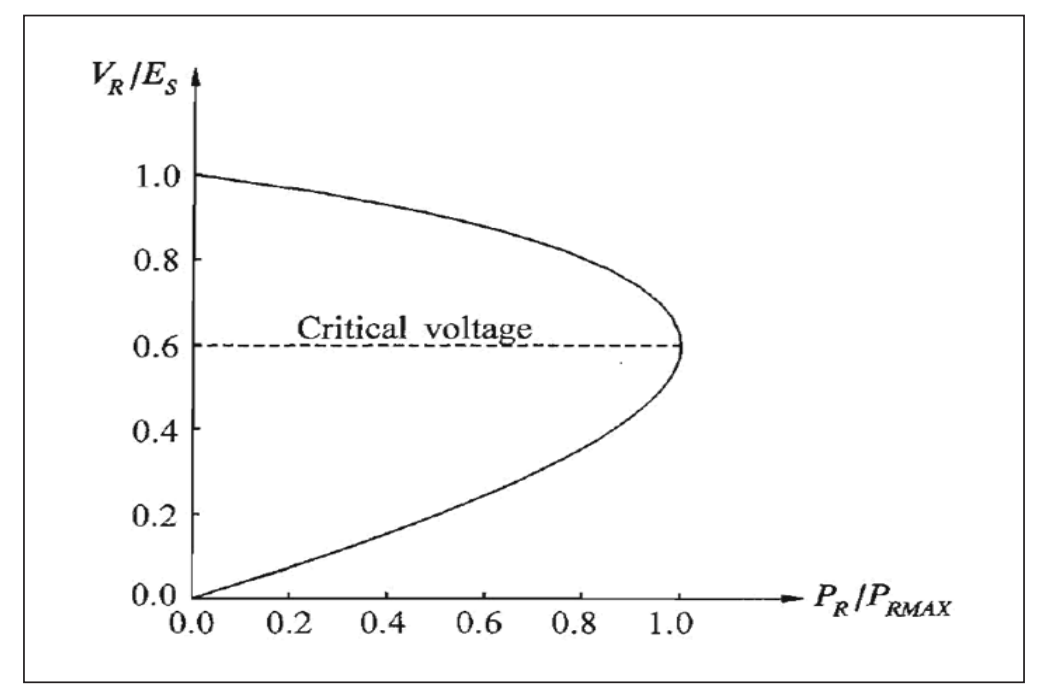

Figure 1. P-V curve (Al Abri et al., 2003) 
of power by varying loads would be unstable. The system shows to be stabilized at voltage and power level minor than the intended value when there is a certain impedance static load characteristics. On the contrary, the system exhibits instability through collapse of the load voltage when there is a certain power load characteristics. With other characteristics, the voltage is determined by the composite characteristic of the transmission line and load. If the load is supplied by transformers with automatic under load tap-changing, the tapchanger action will try to raise the load voltage (Kundur et a., 1994).

Continuation power flow (CPF) is a method to find out some voltage stability conditions of a grid system. It is also used to find out the load margin. At several load quality and load level, the effect in voltage level is observed. Thus, an area is found from voltage vs. load curve where the voltage of a system is collapsed (Ajjarapu \& Christy, 1992).

\section{METHODS}

The following procedure had been conducted for research purpose:

- Examining the impact of solar PV integration on IEEE 14-bus system.

- Analyzing the variation of loadability $(\lambda)$ for placement and fraction of PV plant in IEEE 14 bus system condition

- Finding the maximum limit of PV integration for each bus for IEEE 14-bus system

- Examining the interaction between Photovoltaic and induction motor in an electrical grid.

For the analysis purpose, Power System Analysis Toolbox (PSAT) simulation software was used. From PSAT a test system was chosen named d_014.mdl bus system which was derived from IEEE14-bus system. Figure 2 is the 14 bus PSAT generalized test model.

Generalized IEEE 14-bus system consists of 14-buses including the slack bus. The rating of these buses varies from $69 \mathrm{KV}$ to $13.8 \mathrm{KV}$. Bus 1 to bus 5 is garish in color because this color signifies high voltage and power rating of $69 \mathrm{KV}$ and 100MVA where rest of the buses (except bus-8) is blue in color signifying comparatively medium voltage of $13.8 \mathrm{KV}$. Bus number-8 is unique because it is the only bus of rating $18 \mathrm{KV}$ the color these buses is typically green. There are other components like transmission lines and step-up or stepdown transformers connecting and establishing a network of fourteen buses. Apart from these, there are two static synchronous compensators, one is in bus- 8 another is in bus- 6 .

Static synchronous compensator: Static synchronous compensator (STATCOM) also called Static synchronous condenser is a regulating device used on alternating current electricity transmission networks. It is based on a power electronics voltage-source converter and can act as either a source or sink of reactive AC power to an electricity network. If connected to a source of power, it can also provide active AC power. Usually, a STATCOM is installed to support electricity networks that have a poor power factor and often poor voltage regulation. There are, however, other uses, the most common use is for voltage stability. 
Slack bus: In electrical power systems a slack bus (or swing bus), defined as a (V $\delta$ ) bus, is used to balance the active power $|\mathrm{P}|$ and reactive power $|\mathrm{Q}|$ in a system while performing load flow studies. The slack bus is used to provide for system losses by emitting or absorbing active and/or reactive power to and from the system.

Power for slack bus $=$ Total Power going into the system - Total Power going out of the System + Transmission line losses

The slack bus is the only bus for which the system reference phase angle is defined. From this, the various angular differences can be calculated in the power flow equations. If a slack bus is not specified, then a generator bus with maximum real power $|\mathrm{P}|$ acts as the slack bus. A given scheme can involve more than one slack bus.

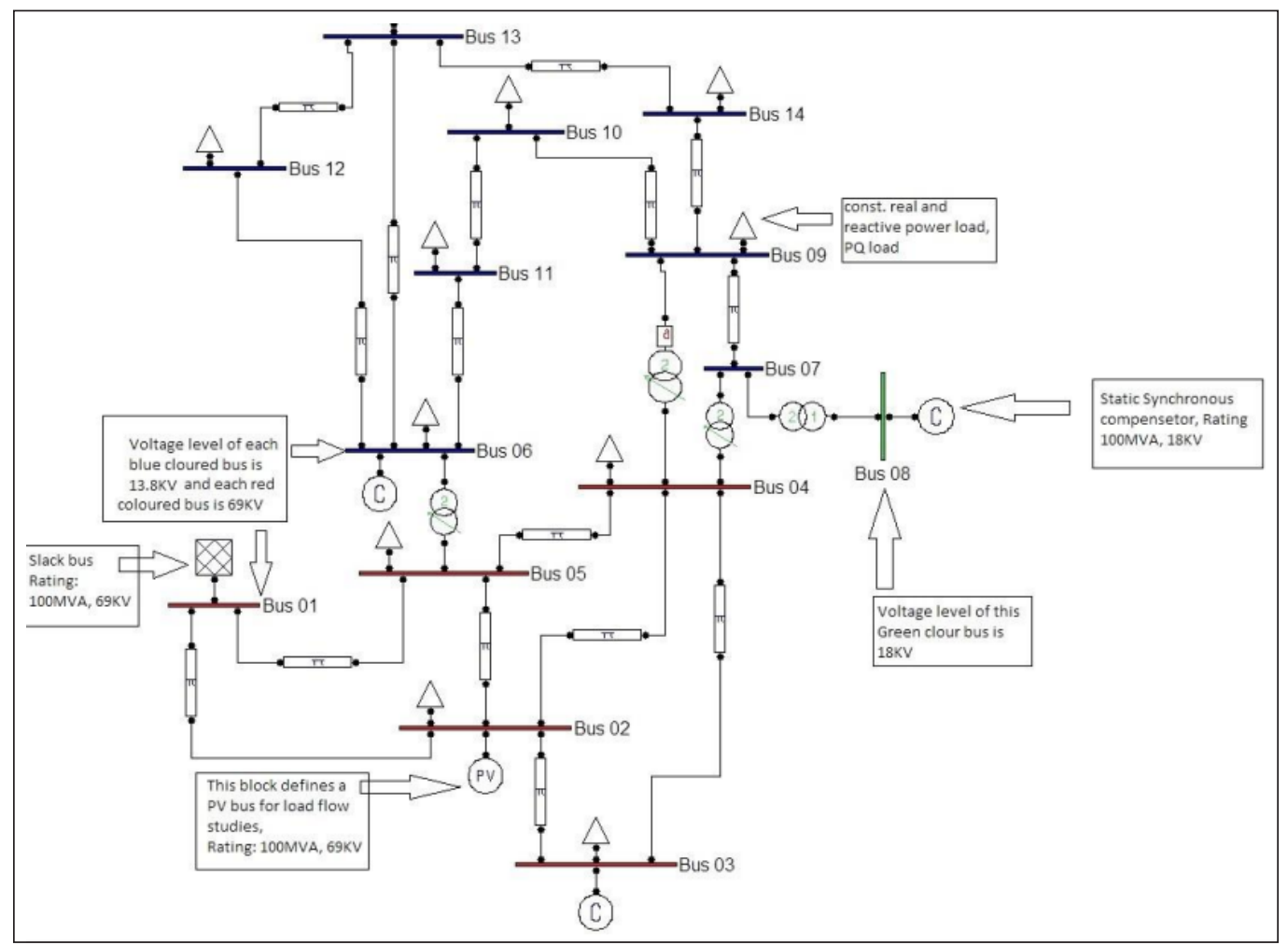

Figure 2. IEEE 14-bus PSAT model

\section{RESULTS AND DISCUSSION}

Load ability of IEEE 14-bus PSAT model varies with placement of PV plant at different buses. Load ability also varies with the size of a single generation plant and total number of generation plant after integrating different buses. 
Value of loadability is represented by value of lamda $(\lambda)$ in the simulation. The load ability of the default system which is taken from PSAT is $\lambda=2.8286$.

Table 1 shows the results of loadability due to placement of solar PQ generator for every individual bus for different cases have been presented. In those cases, solar PQ generator of rating 30MW of active power and 0 MVAR of reactive power were placed at one bus for different time from bus number 2 - 14. Bus number-1 is slack bus, so no generator is added here.

In Figure 3, 14 bus default is the system where no extra generation unit was used. It was taken directly from PSAT without any modification. Table 1 shows that load ability increases (compared to 14 bus default) after integration of solar PQ generator, except at bus number 2, 6, 3, and 8 where load ability decreases. It is due to constant PV generator at bus 2 and synchronous condenser at bus number 3, 6 and 8 . The increase rate at other buses is not same although the same size generator is placed.

For fractioning effect, size of single generation plant and number of total generation plant were changed maintaining same total generation capacity. In some cases, multiple

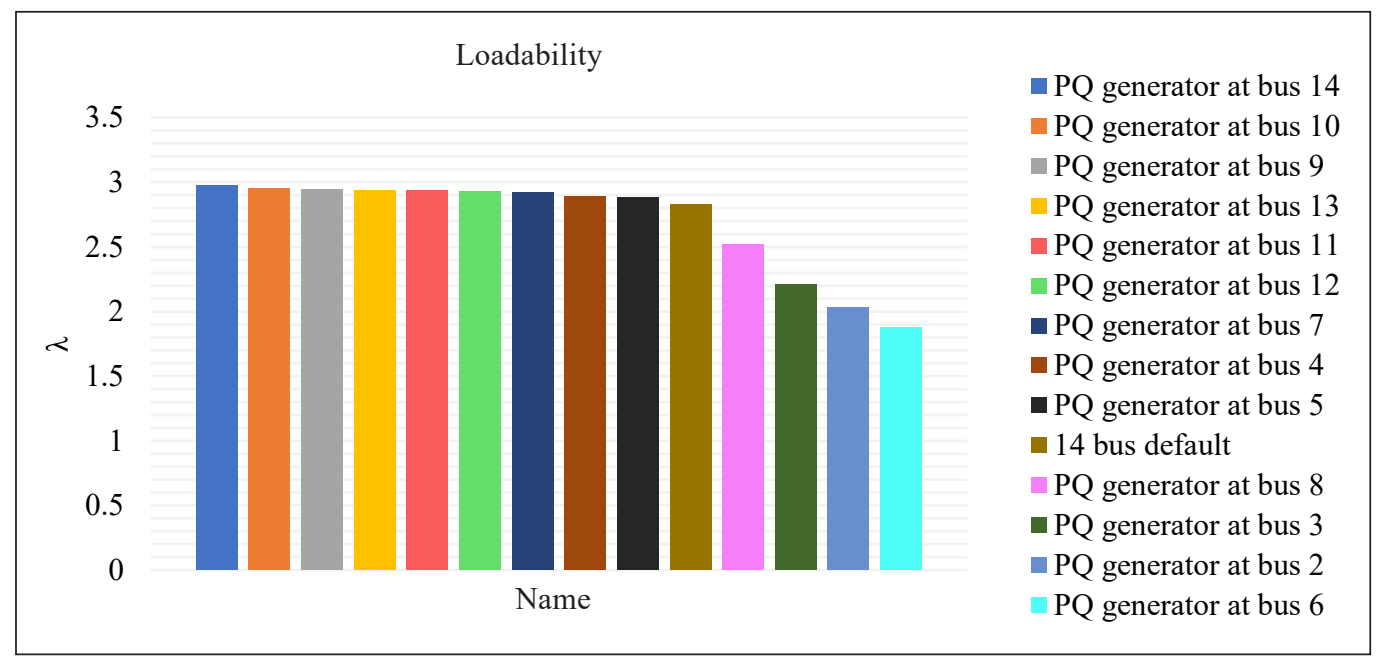

Figure 3. Variation of Load ability due to placement of PV generation unit for different buses

Table 1

Variation of loadability due to placement of the sized generation unit for various buses

\begin{tabular}{lc}
\hline $\begin{array}{l}\text { Generation unit installed bus } \\
\text { number Name }\end{array}$ & $\begin{array}{c}\text { Loadability } \\
(\lambda)\end{array}$ \\
\hline PQ generator at bus 14 & 2.9755 \\
PQ generator at bus 10 & 2.9484 \\
PQ generator at bus 9 & 2.9453 \\
PQ generator at bus 13 & 2.9379 \\
PQ generator at bus 11 & 2.9346 \\
PQ generator at bus 12 & 2.9291 \\
PQ generator at bus 7 & 2.9241 \\
PQ generator at bus 4 & 2.8928 \\
PQ generator at bus 5 & 2.8801 \\
14 bus default & 2.8286 \\
PQ generator at bus 8 & 2.5175 \\
PQ generator at bus 3 & 2.2129 \\
PQ generator at bus 2 & 2.0344 \\
PQ generator at bus 6 & 1.8792 \\
\hline
\end{tabular}




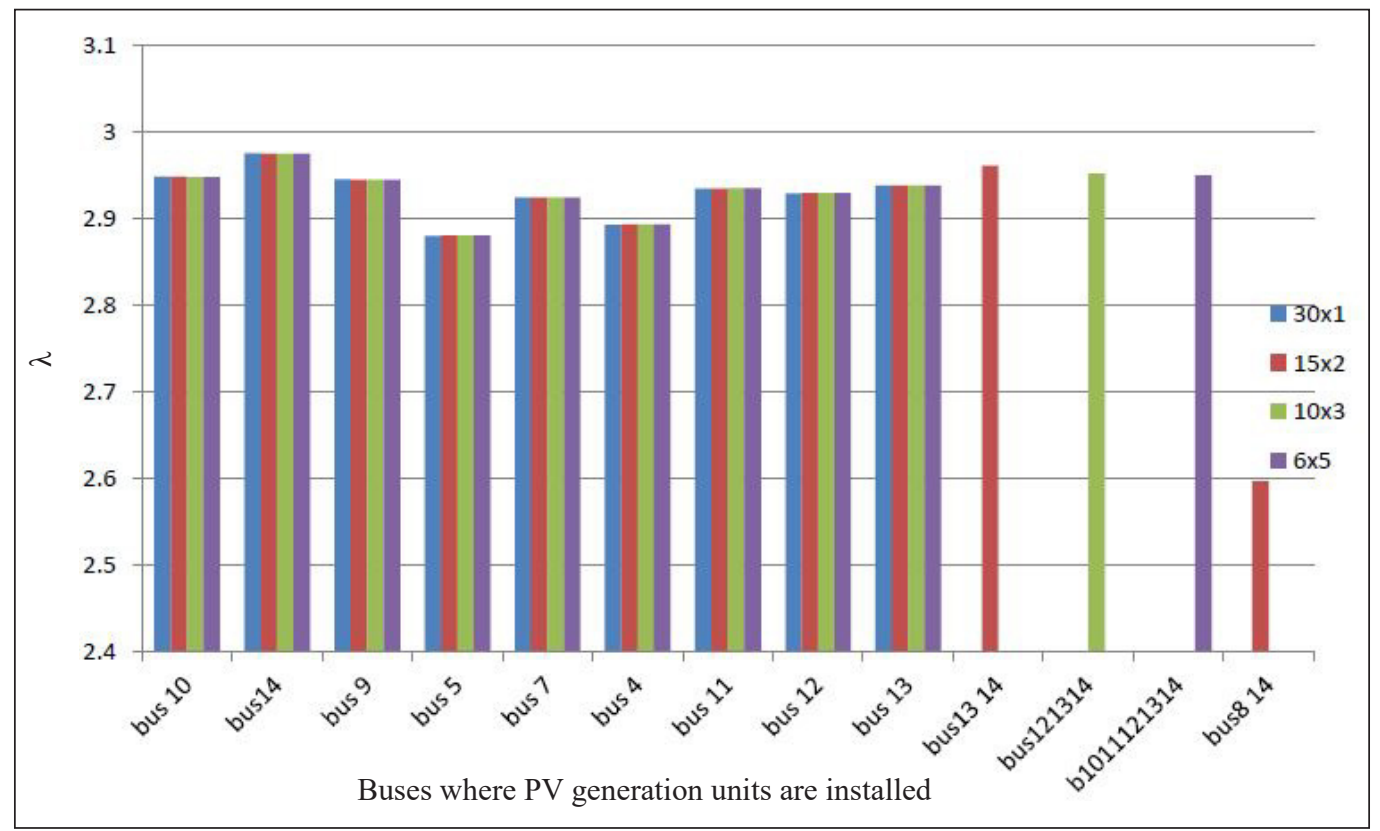

Figure 4. Variation due to fractioning and placement of generation unit at different buses

generation plants were integrated at same buses and in other cases plants were distributed at different buses. Effect of fractioning and placement of generation unit at different buses are shown in Figure 4.

As can be observed, the fractioning effect on load ability by chronological order was $\mathrm{B} 14$ (bus 14) $=\mathrm{B} 10=\mathrm{B} 09>\mathrm{B} 12=\mathrm{B} 11>\mathrm{B} 13=\mathrm{B} 07>\mathrm{B} 04=\mathrm{B} 05$ (Figure 4). Generally, for integration of a new PV generation plant, the buses which exhibit increasing load are also sensitive to the fractioning generation unit. The three buses (bus 14, bus 10 and bus 9), where loadability was higher as compared to others, if we did fractioning in generation on those three buses, loadability decreased. Where on other buses, loadability increased if generation unit was fractioned. In case of distribution at different buses load ability decreased with increase of plant number.

The three buses (bus 14, bus 10and bus 9), where loadability was higher as compared to others, if we did fractioning in generation on those three buses, loadability decreased. Where on other buses, loadability increased if generation unit was fractioned. In case of distribution at different buses, load ability decreased with increase of plant number.

In this case solar PQ generation unit was integrated at different buses of IEEE 14-bus system in PSAT. Ratings of the solar generation unit were increased until system became unstable. In each simulation power factor of PQ generation unit was considered 0.94.

In the Table 2, maximum input power was tested for each bus for integration PQ generation unit. For the bus number 5, active power limit was found maximum (i.e. 1198.5 MW) 
Table 2

Maximum limit for input power of integrated PV for each bus

\begin{tabular}{lcc}
\hline Bus number loadability & Max. Active Power (MW) & Max. Reactive Power (MVAR) \\
\hline Bus 5 & 1198.5 & 76.5 \\
Bus 4 & 1132.7 & 72.3 \\
Bus 7 & 526.4 & 33.6 \\
Bus 9 & 437.1 & 27.9 \\
Bus 10 & 352.5 & 22.5 \\
Bus 11 & 329 & 21 \\
Bus 12 & 291.4 & 18.6 \\
Bus 13 & 258.5 & 17.5 \\
Bus 14 & 253.8 & 16.2 \\
\hline
\end{tabular}

Figure 5 shows that Maximum limit is higher for bus which is closer to the slack bus. As the distance rose from slack bus the limit decreased. Significant change occurred in maximum limit from bus 4 to bus 7 due to change in bus voltage level from $69 \mathrm{KV}$ to 13.8 $\mathrm{KV}$. The voltage level of slack bus and bus number 2, 3, 4 and 5 was $69 \mathrm{KV}$ where the voltage level of the rest of the buses was $13.8 \mathrm{KV}$ except bus 8 where the voltage level was $18 \mathrm{KV}$.

To examine the interaction between photovoltaic plant and induction motor, solar PQ generation plant was integrated along with induction motor at different buses. The effect on load ability due to integration of any motor alongside PV generation plant was noted and is shown in the Table 3 and Figure 6. In this case induction motor rating was 3MVA, $11 \mathrm{KV}$ and $50 \mathrm{~Hz}$. Solar PV generation unit was also placed, and the rating was increased gradually, and the effect was monitored.

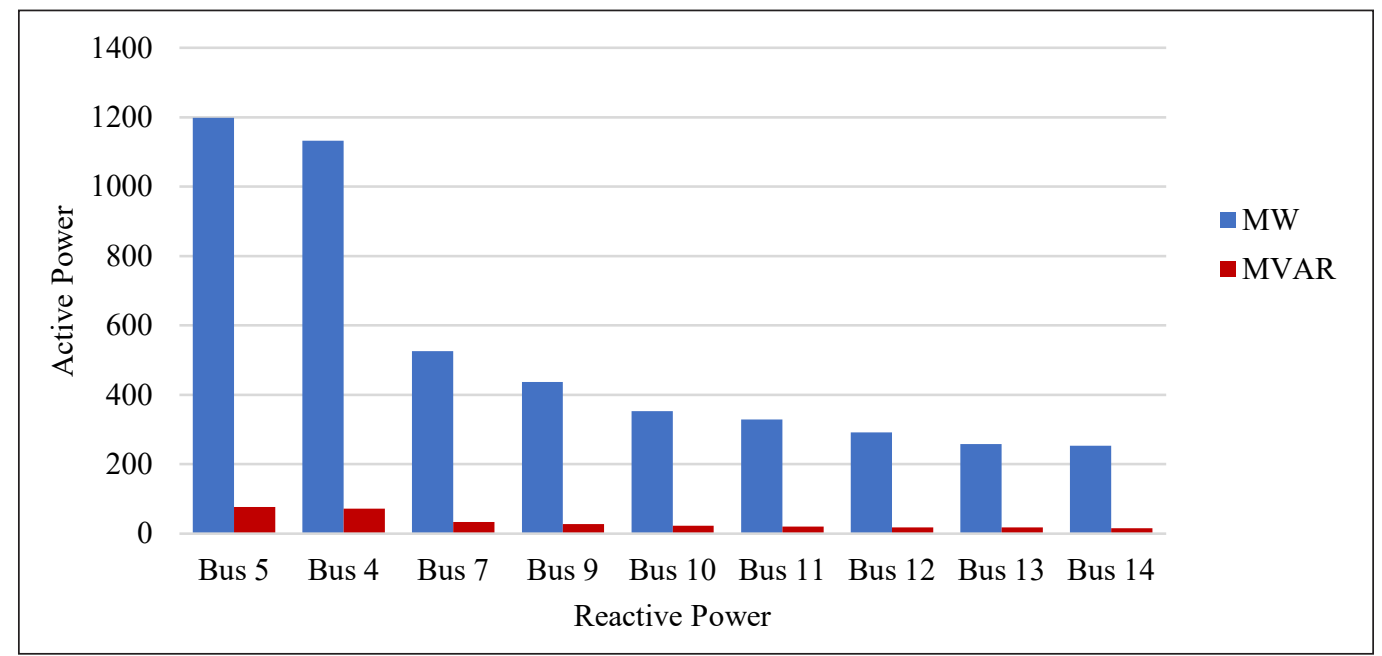

Figure 5. Maximum real power and reactive power limit 
Table 3

Effect on loadability due to integration of induction motor and PV generation plant

\begin{tabular}{lc}
\hline Name & Loadability \\
\hline 14-bus default file & 2.8286 \\
14-bus default file & 2.8286 \\
Induction_Motor_Without_PQ Generation & 1.8676 \\
Induction_Motor_PQ1MWatB14 & 1.8695 \\
Induction_Motor_PQ2MWatB14 & 1.8918 \\
Induction_Motor_PQ3MWatB14 & 1.8938 \\
Induction_Motor_PQ4MWatB14 & 1.875 \\
Induction_Motor_PQ5MWatB14 & 1.8768 \\
Induction_Motor_PQ6MWatB14 & 1.8996 \\
\hline
\end{tabular}

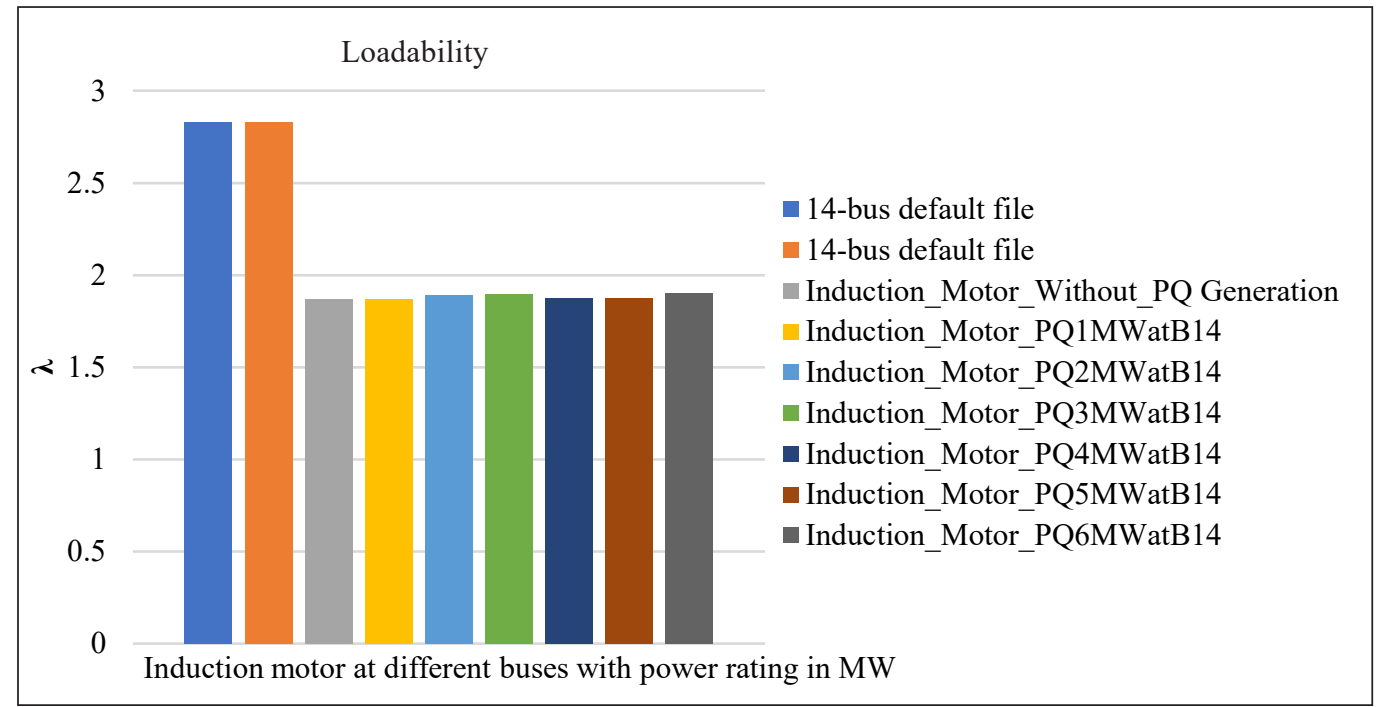

Figure 6. Effect on load ability due to integration of induction motor and PV generation plant

Effect of fractioning of induction motor and interaction with solar PQ generation plant: Table 4 and Figure 7 describe six induction motors of 0.5MVA were integrated with the bus. Rating of PQ generation unit was increased gradually from $1 \mathrm{MW}$ to $6 \mathrm{MW}$.

Load ability was increased if the PQ generator was fractioned. Stability was better if the induction motor was fractioned. Loadability increased linearly with increase of rating of solar PQ generator in the case of fractioned motor. On the other side, load ability increased exponentially up to $3 \mathrm{MW}$ of solar PQ generator integration.

Modeling of solar photovoltaic integrated medium voltage distribution system was done using PSAT simulation tools. To find out the placement and fractioning effect of solar plants the placements of solar photovoltaic plant at different buses had been done. 
Table 4

Comparison between single induction motor and multiple induction motor connected to bus 14

\begin{tabular}{lcc}
\hline Rating of PQ gen & Lodability for single induction motor & Lodability for multiple induction motor \\
\hline without pq gen & 1.8676 & 1.9254 \\
PQ1MW & 1.8695 & 1.9355 \\
PQ2MW & 1.8918 & 1.9396 \\
PQ3MW & 1.8938 & 1.944 \\
PQ4MW & 1.875 & 1.9465 \\
PQ5MW & 1.8768 & 1.9514 \\
PQ6MW & 1.8996 & 1.9562 \\
\hline
\end{tabular}

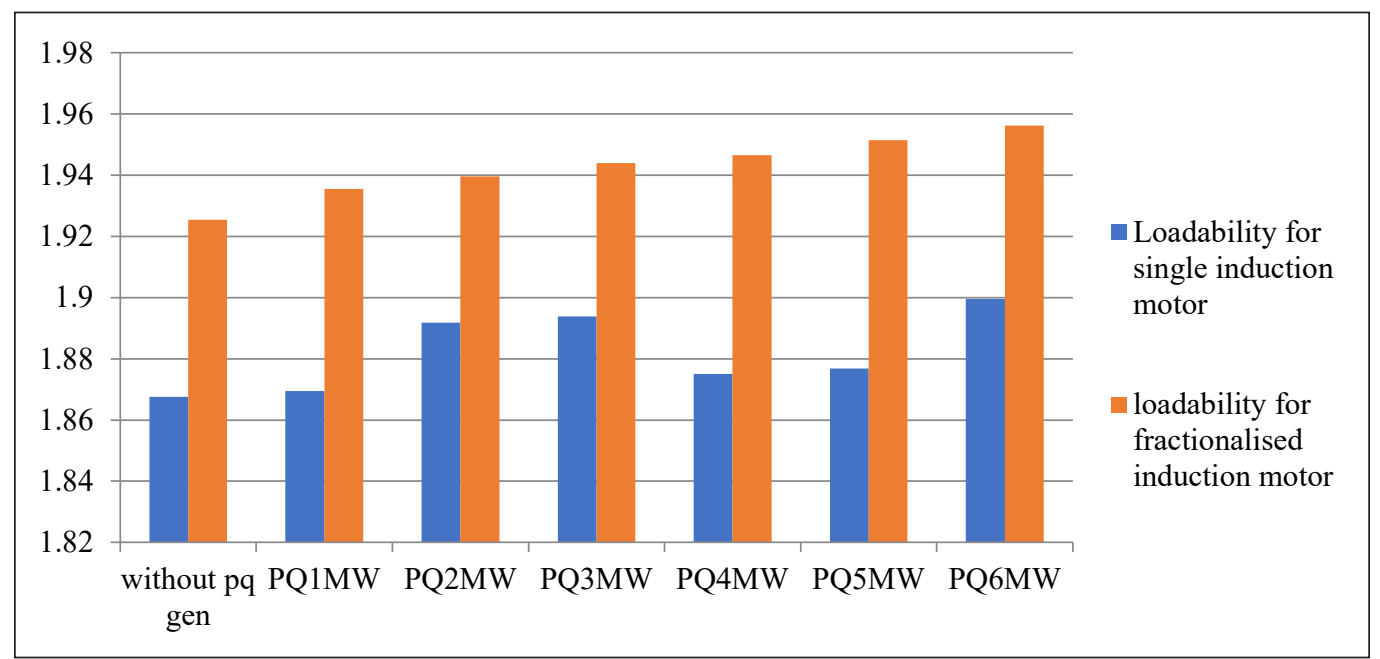

Figure 7. Comparison between single induction motor and multiple induction motor connected system.

\section{CONCLUSION}

It was found that loadability varied with fractioning and placement of solar plant at different buses. Maximum input rating of solar plant for an individual bus was not same for all buses. Loadability increased if the PQ generator was fractioned at bus 14 and stability was better if the induction motor was fractioned.Load ability increased linearly with increase of rating of solar PQ generator in the case of fractioned motor. On the other side, load ability increased exponentially up to $3 \mathrm{MW}$ of solar PQ generator integration. Due to research facility in laboratory the work had not been done exclusively.

The research work needs to be developed in future. Effects of placement $\&$ fraction of solar PV generation \& induction motor in loadability can be analyzed using other simulation software for other test systems. 


\section{ACKNOWLEDGEMENT}

This research work was submitted in partial fulfilment of the requirement for the degree of MS in Renewable Energy Technology at Institute of Energy, University of Dhaka. This paper has been put into the digital library (https://repository.library.ac.bd).

\section{REFERENCES}

Ajjarapu, V., \& Christy, C. (1992). The continuation power flow: A tool for steady state voltage stability analysis. IEEE transactions on Power Systems, 7(1), 416-423. https://doi.org/10.1109/59.141737

Al Abri, R. S., El-Saadany, E. F., \& Atwa, Y. M. (2013). Optimal placement and sizing method to improve the voltage stability margin in a distribution system using distributed generation. IEEE transactions on Power Systems, 28(1), 326-334. https://doi.org/10.1109/TPWRS.2012.2200049

Azmy, A. M., \& Erlich, I. (2005). Impact of distributed generation on the stability of electrical power system. In IEEE Power Engineering Society General Meeting, 2005 (pp. 1056-1063). IEEE Conference Publication. https://doi.org/10.1109/PES.2005.1489354

Begovic, M., Fulton, D., Gonzalez, M. R., Goossens, J., Guro, E. A., Haas, R. W., \& Postforoosh, J. (1995). Summary of system protection and voltage stability. IEEE Transactions on Power Delivery, 10(2), 631638. https://doi.org/10.1109/61.400868

Ingelsson, B., Lindstrom, P. O., Karlsson, D., Runvik, G., \& Sjodin, J. O. (1997). Wide-area protection against voltage collapse. IEEE Computer Applications in Power, 10(4), 30-35. https://doi.org/10.1109/67.625371

Khan, M., Arifin, M., Haque, A., \& Al-Masood, N. (2013). Stability analysis of power system with the penetration of photovoltaic based generation. International Journal of Energy and Power Engineering, 2(2), 84-89. https://doi.org/10.11648/j.ijepe.20130202.18

Kotamarty, S., Khushalani, S., \& Schulz, N. (2008). Impact of distributed generation on distribution contingency analysis. Electric Power Systems Research, 78(9), 1537-1545. https://doi.org/10.1016/j.epsr.2008.01.020

Kundur, P., Balu, N. J., \& Lauby, M. G. (1994). Power system stability and control. McGraw-Hill Inc.

Londero, R. R., Affonso, C. M., \& Nunes, M. V. A. (2009). Impact of distributed generation in steady state, voltage and transient stability-Real case. In 2009 IEEE Bucharest PowerTech (pp. 1-6). IEEE Conference Publication. https://doi.org/10.1109/PTC.2009.5282016

Muhammed, A. O., \& Rawa, M. (2020). A systematic PVQV-Curves approach for investigating the impact of solar photovoltaic-generator in power system using powerworld simulator. Energies, 13(10), Article 2662. https://doi.org/10.3390/en13102662

Rahman, M. M., Barua, S., Zohora, S. T., Hasan, K., \& Aziz, T. (2013). Voltage sensitivity based site selection for PHEV charging station in commercial distribution system. In 2013 IEEE PES Asia-Pacific Power and Energy Engineering Conference (APPEEC) (pp. 1-6). IEEE Conference Publication. https://doi. org/10.1109/APPEEC.2013.6837191

Slootweg, J. G., \& Kling, W. L. (2002). Impacts of distributed generation on power system transient stability. IEEE Power Engineering Society Summer Meeting, 2, 862-867. https://doi.org/10.1109/ PESS.2002.1043465 
